\title{
Mechanical Structure Design of Stress-generating System of Low-frequency Mechanical Spectrum Apparatus
}

\author{
Zheng-Cun ZHOU ${ }^{a,{ }^{*}}$, Jie DU ${ }^{b}$, Hong YANG ${ }^{c}$, Yong-Jian YAN ${ }^{d}$, Su-Yi GU ${ }^{e}$
}

\author{
School of Mechanical and Electronic Engineering, Suzhou Vocational University, \\ Jiangsu, Suzhou, 215104

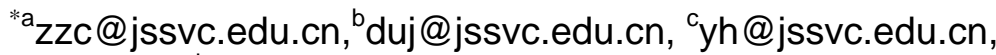 \\ dyyj@jssvc.edu.cn, ${ }^{\mathrm{e}} \mathrm{gsy} @ j s s v c . e d u . c n$
}

\begin{abstract}
Keywords: low-frequency mechanical spectrum apparatus, stress-generating system, Helmholtz
\end{abstract} coils

\begin{abstract}
The shear stress-generating system is designed and calculated in low-frequency mechanical spectrum apparatus to obtain the $10^{-3}$ relative strain for the pure $\mathrm{Al}$ specimen with dimensions $\varnothing 1 \mathrm{~mm} \times 50 \mathrm{~mm}$ in this paper. The physical dimension of Helmholtz coil, the specification of enamel wire and the number of turns as well as the current flow of coil and the specification of power-amplifier source are designed according to the strain value. The acting force between the magnetic field caused by Helmholtz coil and the magnet steel is analyzed. It has been calculated that the diameter of copper enamel wire is $1.12 \mathrm{~mm}$, the number of turns is 1734 , the inner and the external diameter of coil are $40 \mathrm{~mm}$ and $120 \mathrm{~mm}$ respectively and the length is $60 \mathrm{~mm}$. If the load voltage of power-amplifier source is $30 \mathrm{~V}$ and the currency is $4.48 \mathrm{~A}$, the uniform magnetic field of $0.07 \mathrm{~T}$ is produced in the center region of Helmholtz coil and the force applied to the magnet steel will be beyond $26 \mathrm{~N}$.
\end{abstract}

\section{Introduction}

The measurement of low frequency internal friction is carried by using a low frequency internal friction apparatus. A small cyclic stress or an initial stress is applied on the free end of a strip specimen and the amplitude decay (free-decay method) or the phase angle strain lags behind stress (forced-vibration method) is measured and used to characterize the internal friction [1-3]. Magalas et al [4] developed a high-resolution mechanical spectroscopy.

The cyclic or initial stress is produced using Helmholtz coils with electricity and a stable cycle magnetic field is formed between the two coils. A cycle magnetic field is formed when the electric flow with different frequencies is applied on the coils. The interaction between the magnetic field and the magnetic steels installed on the upright pendulum rod will produce the cycle stress [2]. By means of Helmholtz coils, high performance very low frequency forced pendulum was manufactured [2]. Helmholtz coils consist of two coils in series that have the same diameter and number of turns. The direction of electric flow in two coils is the same. Helmholtz coils can produce either an extremely small magnetic field or extremely strong magnetic field with hundreds of gauss. The total magnetic field two current-carrying coils produce is well-distributed within larger scope in the vicinity of axle center when the distance between the two coils is equal to the radius of the coil. Small strain that is often less than $10^{-4}$ was mainly used in the previous internal friction measurement [5-6]. To meet the needs of the development of material science, especially the viscoelasticity measurement of polymers, large strain is also required. Therefore, the strain is increased to $10^{-3}$ in the design of stress-generating system of low-frequency mechanical spectrum apparatus and the design is discussed and described in this paper.

\section{The Calculation of $\tau$ (the Applied Stress on the Specimen) and F (the Acting Force between the Magnetic Field and Magnetic Steels)}

Generally, the strain amplitude is less than $10^{-4}$ for low frequency mechanical spectrum apparatus. To measure the micro-plastic deformation internal friction of materials, the strain amplitude should 
be increased to $10^{-3}$. Therefore, the magnetic field strength must be increased so that the acting force between magnetic field and the magnetic steel is enlarged.

According to Ref. [7], the relative shear strain is equal to strain divided by the length of the specimen, i.e.,

$$
\varepsilon=\frac{\gamma}{l}
$$

Where $\varepsilon$ is the relative shear strain, $\gamma$ is shear strain and $l$ is length of the specimen.

Under the action of pure shear stress $\tau$, the correlation between shear modulus and shear strain are expressed as:

$$
\tau=G \gamma
$$

Where $\tau$ is shear stress applied the specimen, $G$ is shear modulus.

According to Ref. [8], the shear modulus of the rolled pure $\mathrm{Al}$ is $\mathrm{G}=26.5 \mathrm{GPa}$. The diameter of the specimen is often about $1 \mathrm{~mm}$ for large strain measurement. The length of the specimen is selected to be $50 \mathrm{~mm}$. $\tau$ can be calculated to be $1325 \mathrm{MPa}$ when $\varepsilon$ is $10^{-3}$. Therefore, the force of the specimen is calculated to be $\mathrm{F}_{1} \approx 1040 \mathrm{~N}$ for $\varepsilon=10^{-3}$. The calculated force moment in the specimen is $0.52 \mathrm{~N} \cdot \mathrm{m}$ according to Fig. 1 . The force applied on the specimen is originated from the interaction force between the magnetic field and two magnetic steels, which are symmetrically distributed on both sides of upright pendulum rod.

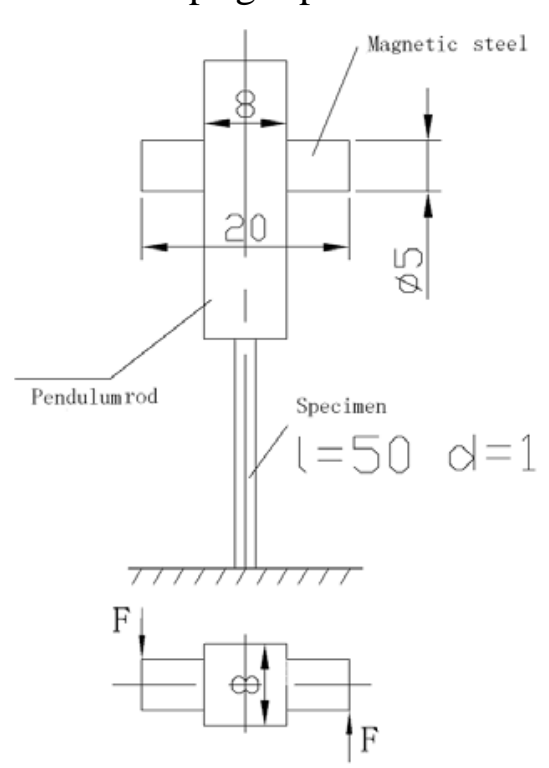

Fig.1 The sketch diagram of acting force of magnetic steel on the specimen (size unit is $\mathrm{mm}$ )

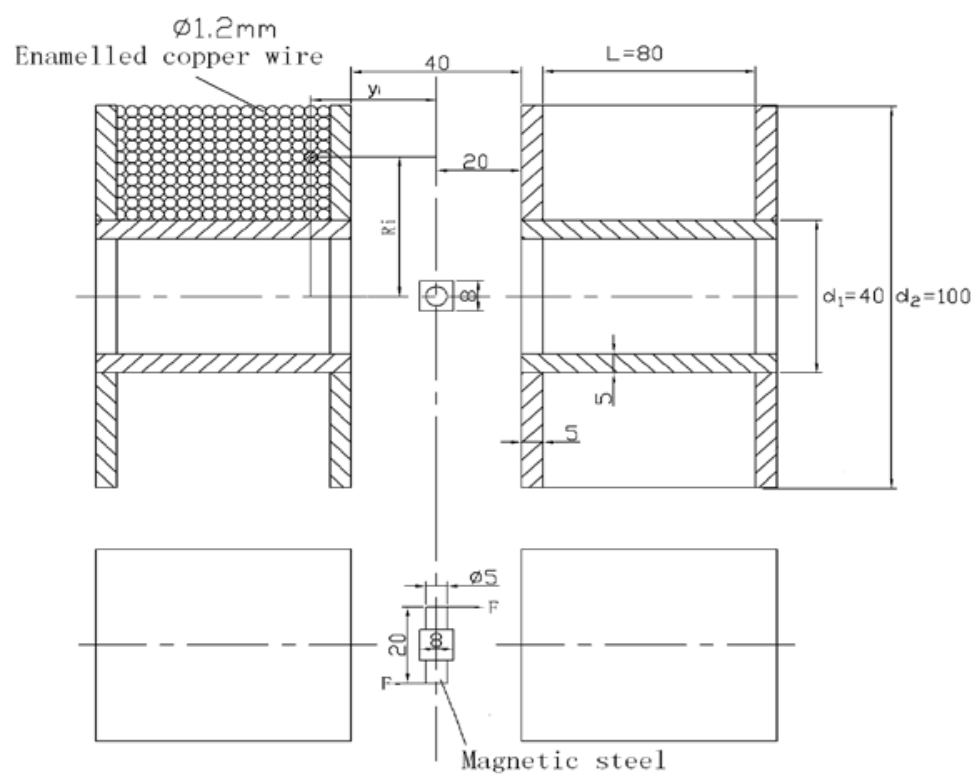

Fig. 2 The position correlation diagram between coils and magnetic steels (size unit is $\mathrm{mm}$ ) 


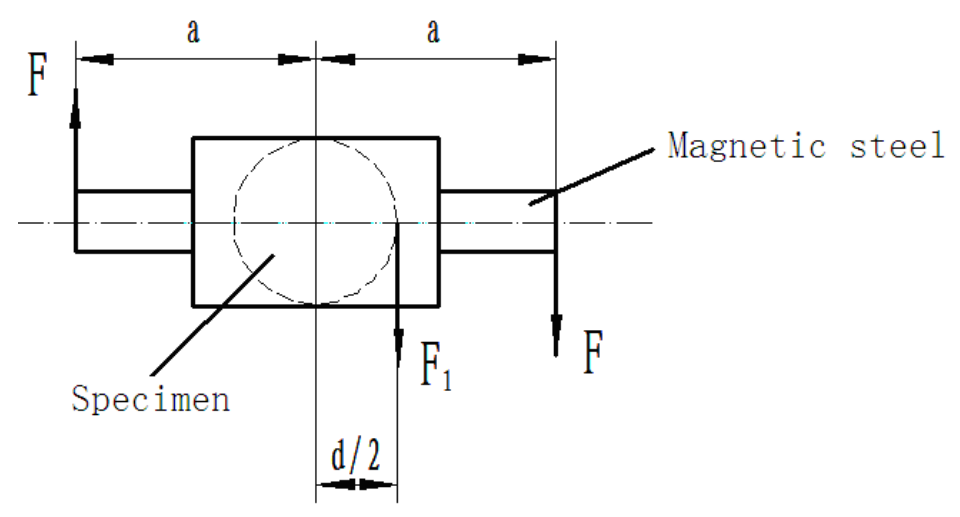

Fig. 3 the force relationship diagram between the magnetic steel and the specimen

According to the principle of equivalent moment of force, the correlation between $\mathrm{F}$ and $\mathrm{F}_{1}$ can be expressed as:

$$
\begin{aligned}
& 2 F \times a=F_{1} \frac{d}{2} \\
& \text { i.e., } F=F_{1} \frac{d}{4 a}
\end{aligned}
$$

$\mathrm{F}$ is the force acting on single magnetic steel. $\mathrm{F}_{1}$ is the force on the specimen, as shown in Fig. 3. $\mathrm{d}$ is the diameter of the specimen in equation (3) and a is the distance from the $\mathrm{F}_{1}$ point to the upright centre of the specimen. If a=10mm, F can be calculated to be about $26 \mathrm{~N}$.

\section{The Design of Helmholtz Coils}

It is well-known that the magnetic field resulted from Helmholtz coils depends many factors, whose magnitude is directly proportion to not only electric current, but also the length of wire in coils [9]. In addition, the magnetic field is correlated to the structure and size of Helmholtz coils. The resistance of wire increases with increasing the wire length and the electric current will be decreased for a given volt. The turns of the coil and the length of wire will increase when the diameter of the wire is decreased for a given structure and size. However, the supported electric current passing the wire will be decreased when the wire is finer, which makes the wire hotter. The resistance in wire will increase. Therefore, many factors should be considered.

According to Refs.[9-10], The magnetic field magnitude (B) at the center between the two coils can be expressed as for Helmholtz with N Turns:

$$
B=\frac{\mu_{0}}{2} \frac{R^{2} N I}{\left(R^{2}+\left(\frac{R}{2}+y\right)^{2}\right)^{3 / 2}}+\frac{\mu_{0}}{2} \frac{R^{2} N I}{\left(R^{2}+\left(\frac{R}{2}-y\right)^{2}\right)^{3 / 2}}
$$

Where $\mu_{0}$ is permeability of vacuum $\left(4 \pi \times 10^{-7} \mathrm{~m} / \mathrm{A}\right)$ and $\mathrm{I}$ is electric current in coils and $\mathrm{R}$ is the radius of the coils. $y$ is the distance between the coil and the center of axis, as shown in Fig. 2 . The enameled wire diameter is $1.2 \mathrm{~mm}$ and the copper wire diameter is $1.12 \mathrm{~mm}$. the cross section of the copper wire is $0.9852 \mathrm{~mm}^{2}$ and $17.8 \Omega / \mathrm{km}$. the size is $\mathrm{d}_{1}=40 \mathrm{~mm}$ and $\mathrm{d}_{2}=100 \mathrm{~mm}(\mathrm{R}=50 \mathrm{~mm})$ and the length is $80 \mathrm{~mm}$ for the present designed Helmholtz coil. The distance between the two coils is $50 \mathrm{~mm}$ when the side plate thickness of the spool is calculated.

$$
R_{n}=1 / 2 d_{1}+1 / 2 d_{\text {copper }}+(n-1) d_{\text {copper }}
$$


If $R_{n}=1 / 2 d_{2}, d_{2}=50 m m, n$ can be calculated to be 26

$\mathrm{y}_{m}=1 / 2 \mathrm{y}+1 / 2 d_{\text {copper }}+(\mathrm{m}-1) \mathrm{d}_{\text {copper }}$

If $\mathrm{y}_{\mathrm{m}}=105 \mathrm{~mm}$, $\mathrm{m}$ can be calculated to be 71

The total turns of a coil is $\mathrm{N}=1846$. The total length can be expressed as:

$$
l_{\text {copper }}=\pi \frac{d_{1}+d_{2}}{2} \times N
$$

Therefore, $l_{\text {copper }}$ can be calculated to be $377176.8 \mathrm{~mm}$ and electric resistance is $6.71 \Omega$. If the volt is $\pm 30 \mathrm{~V}$, the electric current will be about $4.48 \mathrm{~A}$, which is reasonable. Therefore, the nominal volt of the power amplifier is $\pm 35 \mathrm{~V}$ and the nominal current is $\pm 5 \mathrm{~A}$.

According to Ref. [11], the magnetic field is almost distributed in the center region of $|\mathrm{y}| \leq 0.2 R$ along the axis direction and the uniformity of magnetic field is more than $95 \%$ along the radial direction. The $0.2 \mathrm{R}$ is equal to $10 \mathrm{~mm}$ and the turning radius of the magnetic steel is $10 \mathrm{~mm}$ in this design. The magnetic steel is located in the center region of magnetic field of $|y| \leq 0.2 R$. Therefore, this design is reasonable for a practice application.

According to Ref. [9], the magnetic field produced by Helmholtz coils for each turn at the center region can be calculated in follow:

$$
B_{i j}=\frac{\mu_{0} I}{2} \sum_{i=1}^{k} \sum_{j=1}^{n} \frac{R_{i}^{2}}{\left(R_{i}^{2}+y_{j}^{2}\right)^{3 / 2}}
$$

Where Ri and $\mathrm{y}_{\mathrm{i}}$ is the distance between $\mathrm{i}$ turn coil/j turn coil and Helmholtz center respectively, which units are $\mathrm{mm}$ and the unit of $\mathrm{Bij}$ is Tesla $(\mathrm{T})$. $\mathrm{Ri}$ and $\mathrm{y}_{\mathrm{i}}$ can be expressed respectively:

$$
\begin{aligned}
& R_{i}=20+\frac{1}{2} d_{\text {copper }}+(\mathrm{i}-1) \mathrm{d}_{\text {copper }}=20.6+1.2 \times(i-1) \\
& y_{\mathrm{j}}=25+\frac{1}{2} d_{\text {copper }}+(\mathrm{j}-1) \mathrm{d}_{\text {copper }}=25.6+1.2 \times(j-1) .
\end{aligned}
$$

Where the units of $\mathrm{Ri}$ and $\mathrm{y}_{\mathrm{i}}$ are $\mathrm{mm} . \mathrm{k}$ and $\mathrm{n}$ are 26 and 66, respectively. In terms of the above the equations, the magnetic field magnitude at the center of Helmholtz coils can be estimated to be 0.07T. Therefore, the sizes of $d_{1}=40 \mathrm{~mm}, d_{2}=120 \mathrm{~mm}$ and the length $=60 \mathrm{~mm}$ can be applied for a coil.

\section{The Acting Force between the Magnetic Field Produced by Helmholtz and the Magnetic Steel}

A Re-Fe-B material is used in this design, whose parameters of magnetism are listed in table 1 [12]. From table 1, it can be seen that the maximum magnetic energy product is $279(\mathrm{KJ} / \mathrm{m} 3)$. The turns of the Helmholtz coil is 1000 and electric current is 0.5A in Ref. [13]. The Helmholtz coils produce a magnetic field of $0.006 \mathrm{~T}$ and the magnetic steel produces a magnetic field of $0.007 \mathrm{~T}$. A $7.8 \mathrm{~N}$ can be formed at center region of the specimen. The Helmholtz coils and magnetic steels in their structures are similar to those in Ref. [13]. Therefore, it can be deduced that a force of $26 \mathrm{~N}$ at lowest can be obtained. An accurate force calculation is difficult since the action between the magnetic field and magnetic steels is complicated. 
Table 1 Magnetic performance of Ru-Fe-B alloy

\begin{tabular}{ll}
\hline density & $7.45(\mathrm{~g} / \mathrm{cm} 3)$ \\
\hline work temperature & $80-200\left({ }^{\circ} \mathrm{C}\right)$ \\
coercive force & $955(\mathrm{KA} / \mathrm{m})$ \\
maximum magnetic & $279(\mathrm{KJ} / \mathrm{m} 3)$ \\
energy product & \\
residual magnetism & $1.21(\mathrm{~T})$ \\
material & $\mathrm{N} 42$ \\
intrinsic coercive force & $905(\mathrm{KA} / \mathrm{m})$ \\
Curie temperature & $200\left({ }^{\circ} \mathrm{C}\right)$ \\
\hline
\end{tabular}

\section{Conclusions}

The structures and sizes of the Helmholtz coils are designed for a low frequency mechanical spectrum apparatus. The external diameter of the coil is $120 \mathrm{~mm}$ and the inter diameter is $40 \mathrm{~mm}$ and the length is $60 \mathrm{~mm}$. The turns of the coil are 1734. The diameter of the copper wire is 1.12 $\mathrm{mm}$.

The apparatus can produce a maximum shear strain of $10^{-3}$ for a rolled pure $\mathrm{Al}$ specimen with $1 \mathrm{~mm}$ in diameter and $50 \mathrm{~mm}$ in length. A magnetic field of $0.07 \mathrm{~T}$ can be obtained when the work volt is $30 \mathrm{~V}$ and a force of $26 \mathrm{~N}$ at least can be produced.

\section{Acknowledgements}

This work is sponsored by Qing Lan Project of Jiangsu and supported by Research Foundation of Suzhou Vocation University (2013SZDYJ05).

\section{References}

[1]T. S. Ke, The invention of torsion pendulum internal friction apparatus and developments of internal friction study, Advances in Mechanics. 24 (1994)336-350.

[2] I. Gutiérrez-Urrutia, M. L. Nó, E. Carreño-Morelli,B. Guisolan,R. Schaller, J. San Juan, High performance very low frequency forced pendulum,Mater. Sci. Eng. A. 370(2004)435-439.

[3] Z. S. Li, Q. F. Fang, Torsion pendulum method to evaluate the internal friction and elastic modulus of films,Rev. Sci. Instrum. 74(2003)2477-2480.

[4] L.B. Magalas, M. Majewski, Toward high-resolution mechanical spectroscopy HRMS. Resonant frequency-Young's modulus, Solid State Phenomena. 184 (2012) 473-478.

[5] P. Simas, M. Castillo-Rodríguez, M. L. Nó, S. De-Bernardi, D. Gomez, A. Domínguez-Rodríguez, J. San Juan, High temperature internal friction in fine grain and nano-crystalline Zirconia, Solid State Phenomena. 184 (2012) 271-276.

[6] I. Tkalcec, D. Mari and R. Schaller, Anelastic effects of phase decomposition in 14-carat AuAgCu alloy, Solid State Phenomena. 184 (2012) 277-282.

[7]S. P. Yan, Mechanics of Materials, Science Press, Beijing,2012.

[8]The writing group of “Mechanical Design Manual”, Mechanical Design Manual, China Machine Industry Press, Beijing,1989.

[9] Z. G. Zhu, Physics, China Higher Education Press, Beijing,2004.

[10]Y. E. Chen, Y. Y. Zhu, Hermannvon helmholtz magnetic field distribution by MATLAB, Electronic Technology, No.1(2008) 134-136. 
[11]S. X. She, S. J. Zhang, Simple formulas for analyzing magnetic field homogeneity of helmholtz coil helical solenoid and circular loop, College Physics. 18(1999)1-3.

[12]Information on http://detail.1688.com/offerdetail/545755801.html.

[13]E. D. Brandner, B.S. Shivaram, A.Munier, Design and fabrication of an inverted torsion pendulum for internal friction measurements, Meas. Sci. Technol. Vol.6(1995) 310-313. 